\title{
Visually and memory guided saccades in a case of cerebellar saccadic dysmetria
}

\author{
R Kanayama, A M Bronstein, J Shallo-Hoffmann, P Rudge, M Husain
}

MRC Human

Movement and

Balance Unit,

Institute of Neurology,

National Hospital for

Neurology and

Neurosurgery, Queen

Square, London,

WC1N 3BG, UK

R Kanayama

A M Bronstein

J Shallo-Hoffmann

P Rudge

$M$ Husain

R Kanayama

Correspondence to:

Dr Adolfo Bronstein

Received 15 December 1993

and in revised form

4 February 1994.

Accepted 14 February 1994

\begin{abstract}
Saccades under four specific test conditions (visually guided, visually remembered, vestibular remembered, and cervical remembered) were studied in a 38 year old man with ocular dysmetria due to an angioma of the dorsal cerebellar vermis. The aim of the study was to investigate if the saccadic disorder was specific to certain subsets of saccades elicited by different sensory modalities. The experiments showed that initial saccades were equally hypermetric in all four conditions and that final eye position was normal in all memory guided saccade tests. Eye movements differed after the initial saccade, however. Whereas corrective saccades were seen in most visually guided and visually remembered experiments, postsaccadic centripetal drifts were documented in non-visual (vestibular and cervical) remembered saccades. These results indicate that the cerebellar vermis modulates the amplitude of the initial saccade (pulse size of saccadic innervation) independently of the saccadic task. The finding that postsaccadic drift never occurred when saccades were programmed using visual positional information suggests that the
\end{abstract}

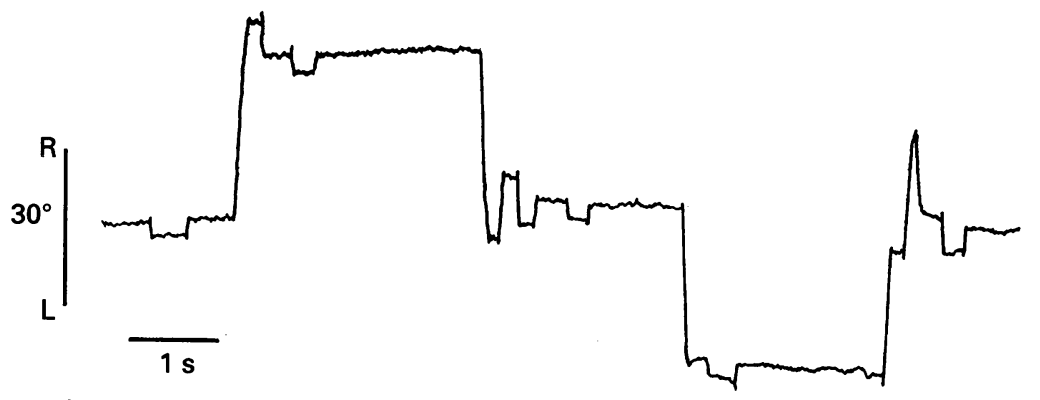

A

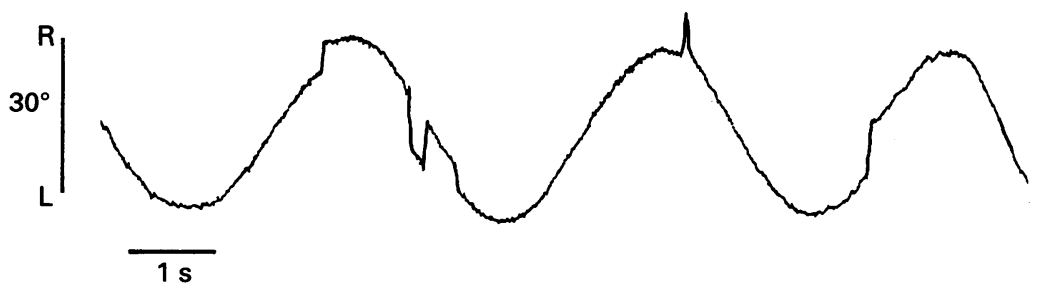

B

Figure 1 (A) EOG recordings during voluntary fixation of targets $\pm 30^{\circ}$ apart Hypermetric saccades are present but there is no gaze evoked nystagmus. (B) Following of a sinusoidally moving laser target at $\pm 17^{\circ}, 0 \cdot 3 \mathrm{~Hz}$. Saccadic intrusions can be seen but slow phase smooth pursuit movements are preserved. dorsal vermis may participate in the process of pulse step integration of saccades elicited by memorised vestibulocervical information.

\section{(F Neurol Neurosurg Psychiatry 1994;57:1081-1084)}

Saccadic dysmetria, in particular hypermetria, is a distinct cerebellar sign. ${ }^{12}$ Both experimental and clinical findings on saccadic eye movements in cerebellar lesions have established that the anterior cerebellar vermis is important for the control of saccadic accuracy..$^{2-4}$ In humans, saccades can be generated in response to a variety of external sensory stimuli (for example, visual, acoustic), be internally generated (voluntary), or generated in response to visual, ${ }^{5}$ vestibular, ${ }^{6}$ or cervical $^{7}$ memorised spatial locations. In neurological disease, the study of these different subtypes of saccades has been particularly fruitful in cerebral cortical or basal ganglia disorders as ocular motor abnormalities have often been found to be task specific. ${ }^{58-10}$ It is therefore surprising that to date no studies have appeared comparing different saccadic paradigms in saccadic dysmetria due to cerebellar lesions. Such is the purpose of the present investigation in a patient chosen for the unusual selectivity of his saccadic dysmetria.

\section{Material and methods \\ CASE REPORT}

A 38 year old man had complained of high tone tinnitus in his left ear for three years and of slurred speech, unsteadiness of gait, and some clumsiness of the right hand for about two years. On examination, he had a moderate bilateral sensory neural hearing loss and was mildly dysarthric. There was no abnormality of tone, power, or sensation but there was some moderate cerebellar ataxia particularly in the right hand and both legs. Clinical and electrooculographic (EOG) examination of the eye movements showed square wave jerks and hypermetric saccades but no spontaneous or gaze evoked nystagmus in the light or darkness, with preserved smooth pursuit and optokinetic nystagmus (fig 1). The horizontal vestibulo-ocular reflex (VOR) assessed by velocity steps $\left( \pm 60^{\circ} / \mathrm{s}\right)$ was symmetric and of normal duration. MRI showed an angioma in the dorsal vermis and the fastigial nuclei area, but the cerebellar hemispheres and the flocculus appeared preserved (fig 2). 
Figure 2 Axial MRI showing an angioma in the dorsal vermis of the cerebellum.

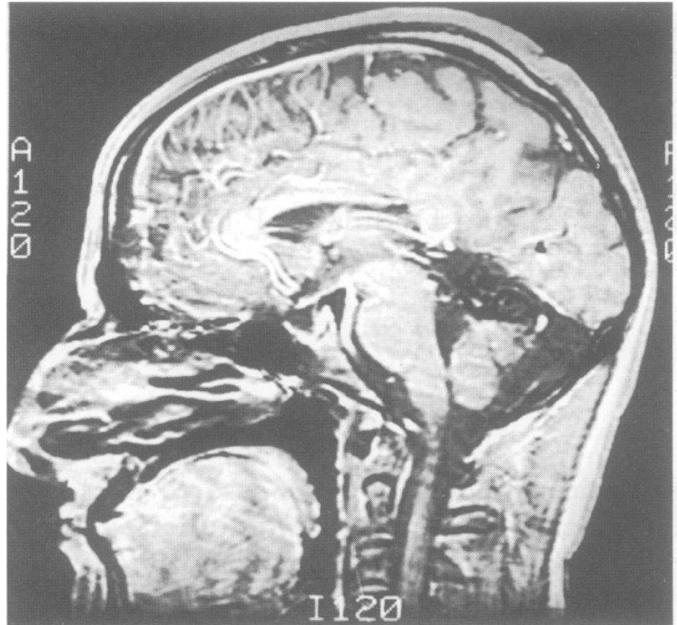

METHODS

Horizontal eye movements were recorded by direct current EOG with bitemporal electrodes. The EOG signal was amplified and digitised at a sampling rate of $250 \mathrm{~Hz}$ and stored on computer for subsequent analysis. Calibrations for $\pm 8,16,24,32$, and $40^{\circ}$ of angular displacement were performed before each test.

Four saccadic tests were performed (visually guided, visual remembered, ${ }^{8}$ vestibular remembered, ${ }^{6}$ and cervical remembered saccades $^{7}$ ) in a light-tight room. During all test conditions, the subject's face and eyes and target illumination were monitored with an infrared video camera. Three test sessions were arranged on separate days to control for the effects of fatigue and order of test presentation, which allowed us to analyse over 200 saccades.

\section{Visually guided saccade test}

The patient was seated in a stable chair with the head firmly fixed with a binaural head clamp, facing a projection screen $(220 \times 150$ $\mathrm{cm}$ ) at a distance of $160 \mathrm{~cm}$. A red dot target $\left(0.4^{\circ}\right.$ in visual angle) was projected at eye level on the screen by a computer controlled video projector. The subject fixated the target in the primary position for three seconds and was instructed to follow the target as quickly as possible when it appeared randomly at different horizontal positions $( \pm 5,7 \cdot 5,10,12 \cdot 5$, 15,20 , and $25^{\circ}$ ) as the central fixation target disappeared.

Visually remembered saccade test

The subject firstly fixated the primary position target. He was informed that after one second, a blue dot target $\left(0.4^{\circ}\right.$ in visual angle) would flash $(200 \mathrm{~ms})$ in an unpredictable lateral position $( \pm 5,7 \cdot 5,10,12.5,15,20$, and $\left.25^{\circ}\right)$. The subject was further instructed to maintain fixation on the central dot until it was switched off, 0.8 seconds after the lateral flash. When the central dot was extinguished, the subject was required to look to the "visually remembered" position of the flashed target, in total darkness. Lastly, after the subject had made the saccade (one second after extinction of the central point), he was instructed to fixate a target appearing in the position of the flashed stimulus, so that any error could be measured from the amplitude of the corrective saccade.

\section{Vestibular remembered saccade test}

In essence, this test is an ocular pointing task, based on memorised vestibular information. ${ }^{6}$ The subject was seated in a computer controlled rotating chair (Contraves-Goerz Inc; $120 \mathrm{Nm}$ torque) with the head firmly fixed to the chair by a binaural clamp. Two red light emitting diode targets were used in this test. One was attached to the distal end of a bar fixed to the chair, positioned $70 \mathrm{~cm}$ in front of the subject's eyes (chair fixed target). A second light emitting diode was mounted on the floor at eye level (earth fixed target). Firstly, both the chair and earth fixed targets were simultaneously presented, the second $2-3 \mathrm{~cm}$ further from the subject, in line with primary gaze. The subject was informed that about one second after the earth fixed target was turned off, the chair would rotate towards the right or left. Randomly presented angular amplitudes were $\pm 10,20,30$, or $40^{\circ}$, peak velocity 20 or $30 \%$ s. During chair rotation, the subject was required to keep looking at the chair fixed target. When the chair fixed target was extinguished (one second after stopping the chair), the subject was instructed to look back to the point where the preceding rotation had started - that is, the place where the earth fixed target was located. Finally, the earth fixed target was switched on for refixation.

\section{Cervical remembered saccade test}

This procedure tests the accuaracy of saccades when only cervical information is available. ${ }^{7}$ The apparatus was the same as for vestibular remembered saccades but the subject's head was firmly fixed by a chin rest and a binaural head clamp to a rigid frame mounted on the wall. This arrangement had the effect that, during chair (trunk) rotation, the subject's head remained stationary in space thereby stimulating cervical receptors. Before starting chair rotation, both targets were lit in line with primary gaze for three seconds. About one second after the chair fixed target was turned off, the chair was smoothly rotated, for safety reasons by hand, with displacements of \pm 5 to $35^{\circ}$ and peak velocities from 3 to $45 \%$ s. During rotation of the trunk, the subject fixated the earth fixed target. About one second after stopping the chair, the earth fixed target was turned off and the subject instructed to look in the direction of his trunk or legs - that is, in the direction where the chair fixed target would be placed. Then the chair fixed target was switched on and refixated by the subject.

Chair velocity was transduced with a tachometer. Chair position was derived by digital integration of the tachometer signal. Saccades were excluded if they did not occur within one second after the subject was required to make a saccade. The variables measured in each trial were $(a)$ initial saccade amplitude-that is, amplitude of the first 
Figure 3 Mean gain (saccade amplitude/stimulus amplitude) of the initia saccade (hatched bars) and of final eye position (open bar). Vertical bars are $S D$. VIGS = Visually guided saccade test;

VIRS = visually remembered saccade test, VERS = vestibular remembered saccade test; $C R S=$ cervical remembered saccade.

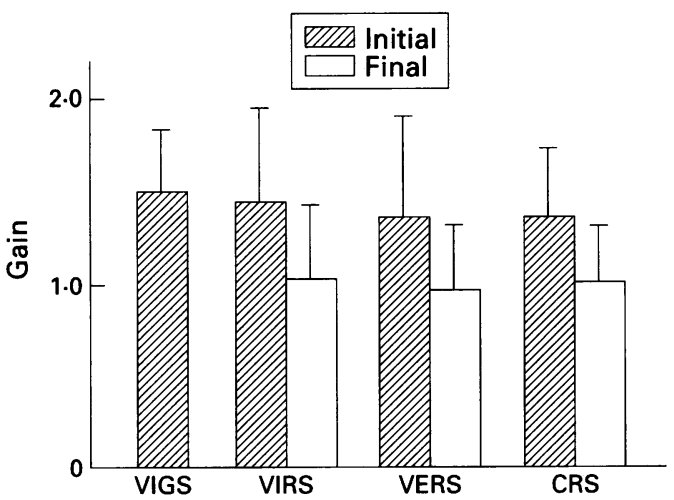

saccade after the subject was required to make a saccade, (b) final eye position-that is, eye position just before the target was reilluminated (except for visually guided saccades as the target remains illuminated throughout). These measurements were expressed as gains - that is, as a ratio to the amplitude of the stimulus. Statistical comparisons were made with Student's $t$ test, $\chi^{2}$ test, and analysis of variance (ANOVA) tests, from the package CSS Statistica (Statsoft).

\section{Results}

Figure 3 shows the mean gain of the initial saccade and final eye position. Results of the three testing sessions and of rightwards or leftwards saccades were pooled as no differences were noted.

Visually guided saccades were hypermetric (gain $>1.0$ ) in both right and left directions regardless of stimulus amplitudes. The mean gain of the initial saccade was 1.52 (fig 3). In most saccades (56 out of $58(97 \%)$ ), corrective saccades towards the target were elicited (fig 4A); the remaining two saccades were normometric.

Visually remembered saccades were also hypermetric (fig 3). The average gain of the initial saccade was 1.46 and that of the final eye position was $1.03(p<0.01)$. In 32 out of 60 saccades $(53 \%)$, there were corrective saccades towards the opposite direction, similar to those seen during visually guided saccades. In the remaining 28 saccades ( $47 \%$ ), eye position after the initial saccade was stationary; eight were normometric, and 20 showed corrective saccades in response to target reillumination as shown in fig $4 \mathrm{~B}$.

Vestibular remembered saccades were hypermetric, with an average gain of initial saccade and final eye position of 1.37 and 0.97 respectively ( $p<0.01$; fig 3 ). In 20 out of 49 saccades $(41 \%)$, centripetal corrective saccades, similar to those occurring in visually guided/remembered saccades, followed the initial hypermetric saccade. In 21 out of 49 saccades (41\%), initial saccades were followed by a post-saccadic centripetal drift, which brought the eye to its final position (fig 4C). The remaining eight saccades were normometric, without a corrective saccade or postsaccadic drift.
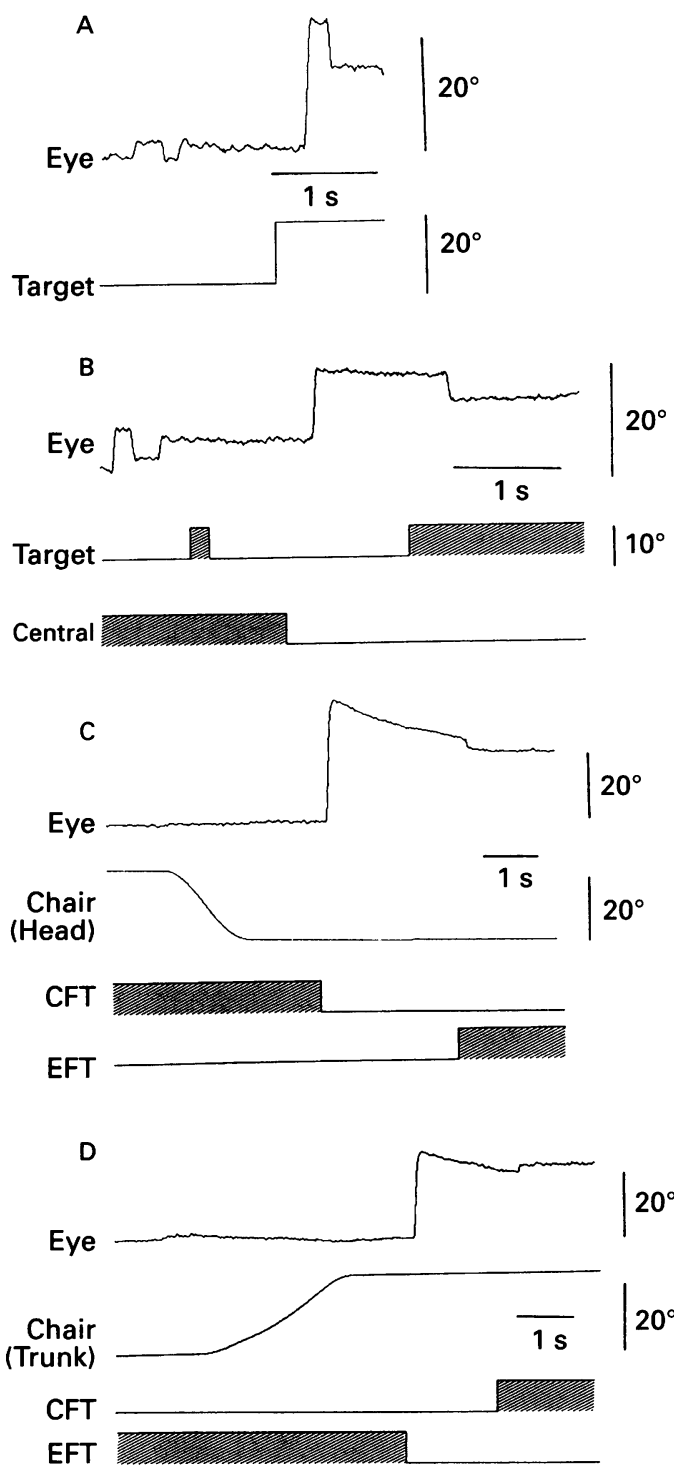

Figure 4 Representative recordings during visually guided $(A)$, visually remembered $(B)$, vestibular guided $(A)$, visually remembered $(B)$, vestibular
remered $(C)$, and cervical remembered $(D)$ saccades. Central = central fixation point; $C F T=$ chair fixed target; $E F T=$ earth fixed target. Hatched areas indicate target illumination.

Cervical remembered saccades were similarly hypermetric. Average initial saccadic gain was 1.37 and final eye position gain was 1.01 ( $p<$ 0.01 ; fig 3 ). Figure $4 \mathrm{D}$ shows a typical recording with the initial hypermetric saccade followed by a postsaccadic drift bringing the eye to its final position with only a slight error. Postsaccadic drift occurred in 21 out of 43 saccades $(49 \%)$. The remaining saccades had either centripetal corrective saccades $(37 \%)$ or were normometric (14\%).

Thus hypermetric initial saccades were present in all four conditions. Initial saccade gains showed no statistical differences among the four conditions. Final eye position gains, which were close to unity, were significantly lower than initial saccade gains in all memory guided saccade tests $(p<0.01)$. The characteristics of the eye movements occurring after the initial saccade-corrective saccades or postsaccadic drift, were significantly related to the saccadic tasks ( $p<0.01, \chi^{2}$ test). 


\section{Discussion}

Cerebellar patients can show different types of ocularmotor abnormalities, depending on the extent and site of the lesion. Lesions of the dorsal vermis or fastigial nucleus tend to cause saccadic dysmetria ${ }^{311}$ whereas lesions of the flocculus and paraflocculus cause abnormalities related to gaze holding and slow phase eye movements, such as gaze evoked, rebound, and down beat nystagmus as well as impaired smooth pursuit, optokinetic nystagmus, and vestibulo-ocular reflex suppression. ${ }^{12}$ In our patient, saccade dysmetria was unusually selective, correlating well with the MRI, which showed that the lesion mainly occupied the dorsal vermis and the fastigial nucleus but spared the flocculus or paraflocculus.

The aim of the experiments was to establish if saccadic dysmetria was dependent on the type of saccadic task. To interpret the findings, however, a brief review of some aspects of the saccade generation process is required (see Leigh and $\mathrm{Zee}^{13}$ and Carpenter ${ }^{14}$ for reviews). Saccades are characterised by high ocular velocity profiles generated by an abrupt rise in the discharge rate of ocular motoneurons. This "burst" or "pulse" of innervation is generated in the paramedian pontine reticular formation. At the end of the pulse, the eyes maintain the newly acquired position in the orbit by a change in the tonic level of neuronal discharge. Such tonic discharge level, often termed the "step", is obtained by a process of mathematical integration of the initial "pulse". The pulse and the step must therefore be matched so that the eyes do not drift after a saccade. As the size of the initial saccade is determined by the initial neural pulse, the dysmetria in this patient accords with the view that vermalfastigial structures are responsible for controlling pulse size. ${ }^{4}$ Thus as our patient showed hypermetria in all experimental paradigms to a similar degree, the findings indicate that these cerebellar structures control pulse size independently of the sensory information, task or mnemonic processes required for the programming of the saccade.

The accuracy in final saccadic eye position in all conditions indicates that this patient's detection of the visual, vestibular, and cervical stimuli was spared. Differences due to test conditions, however, occurred after the initial saccade. Corrective saccades with a stationary eye position between the end of the initial saccade and the corrective saccade were generated in almost all visually induced and visually remembered saccades. In contrast, postsaccadic centripetal drifts were documented during about half of the non-visual tasks ( $43 \%$ of vestibular and $49 \%$ of cervical saccades), a consistent finding not explained by fatigue or order of test presentation.

The process of mathematical integration of the initial saccadic "pulse" into an eye position signal ("step") is carried out by brainstem structures generically termed "the neural integrator". ${ }^{1314}$ The cerebellum is also known to play a part in this process ${ }^{1415}$ probably by providing an integrating loop parallel to the main brainstem integrator. ${ }^{16}$ The rather selective presence of postsaccadic drifts during vestibulocervical remembered saccades in our patient would suggest that the process of either integration or pulse step matching may be different for saccades elicited by different sensory modalities. In cases of visually guided and remembered saccades the tonic step of innervation can be maintained better than saccades programmed on the basis of vestibular or cervical stimulation. The difference may be related to the presence of direct visual positional information in the visually mediated tasks. Position information for the generation of saccades in the non-visual tasks, firstly, has to be reconstructed from dynamic vestibulocervical sensory information ${ }^{17} 18$ and, secondly, has to be transposed from proprioceptive on to visuospatial coordinates. It would be possible that the areas of the cerebellum damaged in our patient, which are known to be both saccade related ${ }^{34}$ and receive significant vestibulocervical projections, ${ }^{19}$ participate in the process of integration for saccades programmed using vestibular and cervical information.

1 Selhorst JB, Stark L, Ochs AF, Hoyt WF. Disorders in cerebellar ocular motor control. I. Saccadic overshoot dysmetria an oculographic, control system and clinicodysmetria an oculographic, control system and

2 Zee DS, Yee RD, Cogan DG, et al. Ocular motor abnormalities in hereditary cerebellar ataxia. Brain 1976;99: malities

3 Ritchie L. Effects of cerebellar lesions on saccadic eye movements. F Neurophysiol 1976;39:1246-56.

4 Optican LM, Robinson DA. Cerebellar-dependent adaptive control of primate saccadic system. $\mathcal{F}$ Neurophysiol 1980;44:1058-76.

5 Pierrot-Deseilligny C, Rivaud S, Gaymard B, Agid Y. Cortical control of memory-guided saccades in man. Exp Brain Res 1991;83:607-17.

6 Bloomberg J, Melvill Jones G, Segal B, Mc Farlane S, Soul $J$. Vestibular-contingent voluntary saccades based on cognitive estimates of remembered vestibular informacognitive estimates of remembered vestibul
tion. Adv Otorhinolaryngol 1988;41:71-5.

7 Nakamura T, Bronstein AM. Perception of neck rotation assessed by 'remembered-saccades'. NeuroReport assessed by

8 Lueck CJ, Tanyeri S, Crawford TJ, Henderson L, Kennard C. Antisaccades and remembered saccades in Parkinson's disease. $f$ Neurol Neurosurg Psychiatry 1990;53:284-88.

9 Kennard C, Lueck CJ. Oculomotor abnormalities in diseases of basal ganglia. Rev Neurol (Paris) 1989;145: 587-95.

10 Tropper J, Melvill Jones G, Bloomberg J, Fadlallah H. Vestibular perceptual deficits in patients with parietal lobe lesions. Acta Otolaryngol (Stockh) 1991;suppl 481:528-33.

11 Bötzel K, Rottach K, Büttner U. Normal and pathological saccadic dysmetria. Brain 1993;116:337-53.

12 Zee DS, Yamazaki A, Butler PH, Gucer G. Effects of ablation of flocculus and paraflocculus on eye movements in primate. $\mathcal{F}$ Neurophysiol $1981 ; 46: 878-99$.

13 Leigh RI, Zee DS. The neurology of eye movements. 2nd ed. Philadelphia: FA Davis, 1991.

14 Carpenter RHS. Movement of the eyes. 2nd ed. London: Pion, 1988.

15 Robinson DA. The effect of cerebellectomy on the cat's vestibulo-ocular integrator. Brain Res 1974;71:195-207. Zee DS, Leigh RJ, Mathieu-Millaire F. Cerebellar control of ocular gaze stability. Ann Neurol 1980;7:37-40.

17 Goldberg JM, Fernández C. Physiology of peripheral neurons innervating semicircular canals of the squirrel monkey. I. Resting discharge and response to constant angular accelerations. $\mathcal{F}$ Neurophysiol 1971;34:635-60.

18 Richmond FJR, Abrahams VC. Physiological properties of muscle spindles in dorsal neck muscle of the cat. $\mathcal{F}$ Neurophysiol 1979;42:604-17.

19 Berthoz A, Llinas R. Afferent neck projection to the cat cerebellar cortex. Exp Brain Res 1974;20:385-401. 